\title{
De Sitter Space as a Computational Tool for Surfaces and Foliations
}

\author{
Maciej Czarnecki ${ }^{1}$, Szymon Walczak ${ }^{2}$ \\ ${ }^{1}$ Katedra Geometrii, Wydział Matematyki i Informatyki, Uniwersytet Łódzki, Łódź, Poland \\ ${ }^{2}$ Katedra Metodyki Nauczania Matematyki, Wydział Matematyki i Informatyki, Uniwersytet Łódzki, Łódź, Poland \\ Email: maczar@math.uni.lodz.pl,sajmonw@math.uni.lodz.pl
}

Received February 2, 2013; revised March 5, 2013; accepted March 21, 2013

Copyright (C) 2013 Maciej Czarnecki et al. This is an open access article distributed under the Creative Commons Attribution License, which permits unrestricted use, distribution, and reproduction in any medium, provided the original work is properly cited.

\begin{abstract}
The set of all spheres and hyperplanes in the Euclidean space $\mathbb{R}^{n}$ could be identified with the Sitter space $\Lambda^{n+1}$. All the conformal properties are invariant by the Lorentz form which is natural pseudo-Riemannian metric on $\Lambda^{n+1}$. We shall study behaviour of some surfaces and foliations as their families using computation in the de Sitter space.
\end{abstract}

Keywords: De Sitter Space; Foliation; Conformal Geometry

\section{Introduction}

One of the topics in the theory of foliations is studying geometric properties of leaves e.g. being totally geodesic, totally umbilical, constant mean curvature etc. Two surveys $[1,2]$ by the first author together with Paweł Walczak and Badura descibe progress of this research in the last years.

Conformal geometry using the Sitter space was studied by Langevin and many of his collaborators. Up to now the most extensive explanation is given in [3].

The idea of representing oriented spheres in the de Sitter space is widely presented in [4] by Langevin and Paweł Walczak. They succesfully applied this method to the theory of foliations. Together with Bartoszek they studied properties of so called canal surfaces i.e. envelopes of one parameter families of spheres (cf. [5,6]).

In the paper below we recall some notions of conformal geometry and extend them to study intersections of spheres. The main application is some partial characterizations of totally umbilical foliations on the hyperbolic space $\mathbb{H}^{n}$ in its conformal ball model.

\section{Space of Spheres}

Every $(n-1)$-dimensional round sphere in the Euclidean space $\mathbb{R}^{n}$ is determined by $n$ coordinates of its center and one positive parameter (radius). A hyperplane

${ }^{*}$ This article was supported by Polish NCN grant No. 6065/B/H03/ 2011/40.

2010 Mathematics Subject Classification: 53C12, 53A05, 53A35, $53 \mathrm{~A} 30,53 \mathrm{C} 50$. is the limit of spheres internally tangent to each other when radius tends to infinity. Anyway, in that case there are many different families of spheres having the same limit hyperplane.

More natural is to consider hyperplanes and spheres on the unit sphere $\mathbb{S}^{n}$. Here all the hyperplanes and spheres are simply round $(n-1)$-spheres. Thus the set of $(n-1)$-spheres and hyperplanes in $\mathbb{S}^{n}$ is endowed with a differential structure of $(n+1)$-dimensional manifold.

It is well known that conformal diffeomorphisms of $\mathbb{S}^{n}$ form the Möbius group $\operatorname{Möb}(n)$ isomorphic to the group $\mathrm{SO}^{+}(1, n)$ consisting of linear transformations with determinant equal to 1 preserving the Lorentz form and orientation. Thus the Lorentz product preserves all the conformal properties.

We shall describe this idea more explicitely.

Consider $(n+2)$-dimensional Lorentz space $\mathbb{L}^{n+2}$, i.e. the space $\mathbb{R}^{1, n+1}$ with the Lorentz form $\langle\cdot \mid \cdot\rangle$ given by

$$
\langle x \mid y\rangle=-x_{0} y_{0}+x_{1} y_{1}+\cdots+x_{n} y_{n}+x_{n+1} y_{n+1}
$$

In $\mathbb{L}^{n+2}$ the canonical basis $\left\{e_{0}, e_{1}, \cdots, e_{n+1}\right\}$ is considered.

For $x=\left(x_{0}, x_{1}, \cdots, x_{n}, x_{n+1}\right) \in \mathbb{L}^{n+2}$ we use the notation

$$
\tilde{x}=\left(x_{1}, \cdots, x_{n}, x_{n+1}\right) \text { and } \hat{x}=\left(x_{1}, \cdots, x_{n}\right)
$$

The terminology comes from the special relativity, so we traditionally say that vectors of positive Lorentz norm (i.e. $\langle x \mid x\rangle>0$ ) are called space-like while those of negative (resp. zero) Lorentz norm are time-like (resp. 
light-like).

On the space-like $(n+1)$-plane $\{0\} \times \mathbb{R}^{n+1}$ the Lorentz form reduces to the standard scalar product $\langle.,$.$\rangle and the$ respective Euclidean norm $\|\cdot\|$.

Definition 1 The sets

$$
\begin{aligned}
& \mathcal{L}=\left\{x \in \mathbb{L}^{n+2} \mid\langle x \mid x\rangle=0\right\} \\
& \Lambda^{n+1}=\left\{x \in \mathbb{L}^{n+2} \mid\langle x \mid x\rangle=1\right\} \\
& S_{\infty}^{n}=\left\{x \in \mathcal{L} \mid x_{0}=1\right\}=\left\{x \in \mathbb{L}^{n+2} \mid\|\tilde{x}\|=1, x_{0}=1\right\}
\end{aligned}
$$

are called respectively: the light cone, the de Sitter $(n+1)$-space, the limit sphere.

Furthemore we denote by $S$ the set of oriented (round) $(n-1)$-spheres contained in $\mathbb{S}^{n}$ which is in fact $S_{\infty}^{n}$.

Proposition 2 Assigning to any $s \in \Lambda^{n+1}$ an intersection $s^{\perp} \cap S_{\infty}^{n}$ is an one-to-one correspondence between $\Lambda^{n+1}$ and $S$.

Proof: Observe that for $s \in \Lambda^{n+1}$ we have

$$
\begin{aligned}
& \|\tilde{s}\|^{2}=1+s_{n+2}^{2} \\
& s^{\perp}=\left\{v \in \mathbb{R}^{n+2} \mid\langle s \mid v\rangle=0\right\}=\left\{v \in \mathbb{R}^{n+2} \mid\langle\tilde{s} \mid \tilde{v}\rangle=s_{0} v_{0}\right\} \\
& s^{\perp} \cap S_{\infty}^{n}=\left\{x \in \mathbb{L}^{n+2} \mid\|\tilde{x}\|=1,\langle\tilde{s}, \tilde{x}\rangle=s_{0}, x_{0}=1\right\}
\end{aligned}
$$

Since in the affine hyperplane $x_{0}=1$ the distance from an $n$-plane $E_{s}:\langle\tilde{s}, \tilde{x}\rangle=s_{0}$ to the origin $(\theta, 1)$ equals

$$
\frac{\left|s_{0}\right|}{\|\tilde{s}\|}<1
$$

hence $E_{s}$ intersects the sphere $\|\tilde{x}\|=1$ along $(n-1)$ sphere what is clearly visible in Figure 1.

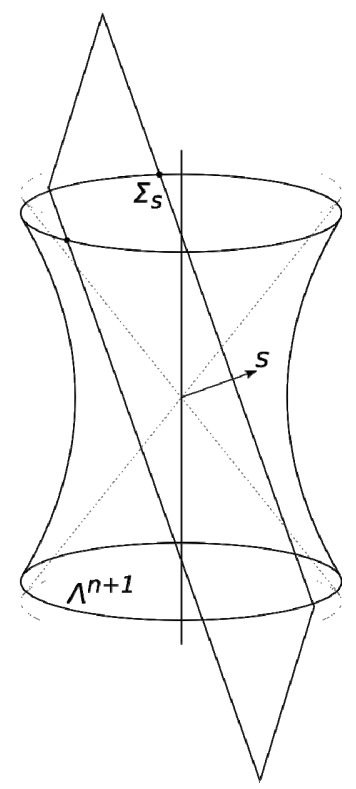

Figure 1. De Sitter space.
The $n$-planes $E_{s}$ and $E_{s^{\prime}}$ (and therefore respective $(n-1)$-spheres) are equal if $s$ and $s^{\prime}$ are linearly dependent. But $\Lambda^{n+1}$ intersects every 1-dimensional vector space $\operatorname{lin}(s), s \in \Lambda^{n+1}$, in exactly two points, so we can think of them as of distinct orientations of the sphere $s^{\perp} \cap S_{\infty}^{n}$.

The above proposition motivates.

Definition 3 Given $s \in \Lambda^{n+1}$ we say that $s$ is the representation of sphere $\Sigma_{s}=s^{\perp} \cap S_{\infty}^{n} \subset \mathbb{S}^{n}$ in the de Sitter $(n+1)$-space.

Since we are interested only in conformal properties of spheres (mainly angle of intersection), we shall proceed with stereographic projection which transforms them conformally onto codimension 1 spheres or affine subspaces.

Proposition 4 Assume that $s, t \in \Lambda^{n+1}$. Then spheres $\Sigma_{s}$ and $\Sigma_{t}$ represented by them:

1) are disjoint if $|\langle s \mid t\rangle|>1$,

2) meet at angle $\alpha \in\left[0, \frac{\pi}{2}\right]$ if $|\langle s \mid t\rangle|=\cos \alpha$.

Proof: The Lorentz form is invariant under Möbius transformations so it is enough to check the statement for spheres (not) meeting a fixed sphere.

Fix the sphere represented by $e_{n+1}$ and observe that $s_{n+1}=\left\langle s \mid e_{n+1}\right\rangle$ for any $s \in \Lambda^{n+1}$. Let $\Pi$ denote the stereographic projection of $S_{\infty}^{n}=\{1\} \times \mathbb{S}^{n}$ onto $\mathbb{R}^{n}=\{1\} \times \mathbb{R}^{n} \times\{0\}$ from the point $-e_{n+1}$.

Thus the projection $\Pi$ is given by the formula

$$
\Pi(z)=\frac{\hat{z}}{1+z_{n+1}}
$$

and its inverse by

$$
\Pi^{-1}(y)=\left(\frac{2 y}{1+\|y\|^{2}}, \frac{1-\|y\|^{2}}{1+\|y\|^{2}}\right), y \in \mathbb{R}^{n} .
$$

It is obvious that $(n-1)$-sphere $e_{n+1}^{\perp} \cap S_{\infty}^{n}$ is invariant under $\Pi$.

For $s \in \Lambda^{n+1}$ we have an equation of $\Pi\left(\Sigma_{s}\right)$

$$
\left\langle\left(\hat{s}, s_{n+1}\right),\left(\frac{2 y}{1+\|y\|^{2}}, \frac{1-\|y\|^{2}}{1+\|y\|^{2}}\right)\right\rangle=s_{0}
$$

or equivalently

$$
2\langle\hat{s}, y\rangle-\left(s_{n+1}+s_{0}\right)\|y\|^{2}+s_{n+1}-s_{0}=0 .
$$

Hence two cases occur:

1) If $s_{n+1}+s_{0}=0$ then $\Pi\left(\Sigma_{s}\right)$ is a hyperplane

$$
\langle\hat{s}, y\rangle+s_{n+1}=0,
$$

2) If $s_{n+1}+s_{0} \neq 0$ then $\Pi\left(\Sigma_{s}\right)$ is the sphere 


$$
\left\|y-\frac{\hat{s}}{s_{n+1}+s_{0}}\right\|=\frac{1}{\left|s_{n+1}+s_{0}\right|} .
$$

For the angle of intersection we obtain:

1) In case of a hyperplane $\|\hat{s}\|=1)$ so $\Pi\left(\Sigma_{s}\right)$ meets the unit sphere at angle $\alpha \in\left[0, \frac{\pi}{2}\right]$ if the Euclidean distance of the center from $\Sigma_{s}$ equals $\cos \alpha$. Thus we have

$$
\cos \alpha=\frac{\left|s_{n+1}\right|}{\|\hat{S}\|}=\left|s_{n+1}\right| .
$$

2) If $\Pi\left(\Sigma_{s}\right)$ is a sphere then it meets the unit sphere at angle $\alpha$ if at any point of intersection the angle at this point in the triangle formed by centers is exactly $\alpha$. Equivalently, by law of cosines

$$
\left\|\frac{\hat{s}}{s_{n+1}+s_{0}}\right\|^{2}=1+\frac{1}{\left(s_{n+1}+s_{0}\right)^{2}}-\frac{2}{\left|s_{n+1}+s_{0}\right|} \cos \alpha
$$

which could be simplified to

0

$$
\begin{aligned}
& =1-\|\hat{s}\|^{2}+s_{n+1}^{2}+s_{0}^{2}+2 s_{n+1} s_{0}-2\left|s_{n+1}+s_{0}\right| \cos \alpha \\
& =2\left(s_{n+1}\left(s_{n+1}+s_{0}\right)-\left|s_{n+1}+s_{0}\right| \cos \alpha\right)
\end{aligned}
$$

or directly $s_{n+1}^{2}=\cos ^{2} \alpha$.

\section{Example 5}

1) 2-dimensional de Sitter space $\Lambda^{2}$ is one-sheeted hyperboloid in 3-dimensional space. Points of $\Lambda^{2}$ represent 0 -dimensional oriented spheres on the unit circle. Thus they are arcs of this circle. According to Prop. 4

a) these arcs are disjoint if the Lorentz product of their representations is $>1$,

b) they are nested if the product is $<-1$,

c) they are knotted if the product is between -1 and 1 .

Excluding the case of representing the same unoriented sphere the product equal -1 means that one arc is include-ed in another, while 1 is the condition for having exactly one point in common.

2) Oriented circles (i.e. discs) on $\mathbb{S}^{2}$ are represented in $\Lambda^{3}$. The circles have 2 (resp. 1,0 ) common points provided that the absolute value of their Lorentz product is in $(0,1)$ (resp. 1, >1).

3) 2-dimensional spheres on $\mathbb{S}^{3}$ are tangent if their representations on $\Lambda^{4}$ have the Lorentz product equal \pm 1 . They intesect along a circle if the product is between -1 and 1 . Otherwise they are nested.

\section{Application to Foliations}

\subsection{Umbilical Foliations of $\mathbb{H}^{n}$}

Hyperbolic $n$-space $\mathbb{H}^{n}$ has the Poicaré ball model which is the unit $n$-ball with conformally changed Euclidean metric. At every point $x$ the Euclidean Riemannian metric is multiplied by $\frac{4}{\left(1-\|x\|^{2}\right)^{2}}$. The ball model is conformal so angles at $\mathbb{H}^{n}$ are preserved.

Complete totally umbilical hypersurfaces in the Euclidean space are spheres and hyperplanes. Conformality of the ball model implies the same is true for $\mathbb{H}^{n}$. Thus every totally umbilical hypersurface in the ball model is intersection of $\mathbb{H}^{n}$ with a (Euclidean) sphere or a (Euclidean) hyperplane.

If we denote by $\partial \mathbb{H}^{n}$ the ideal boundary of $\mathbb{H}^{n}$ which is in fact $(n-1)$-sphere then spheres orthogonal to $\partial \mathbb{H}^{n}$ contain totally geodesic hypersurfaces. Moreover, every sphere making angle $\alpha$ with $\partial \mathbb{H}^{n}$ contains a hypersurfaces with all principal curvatures equal to $\cos \alpha$ sharing ideal boundary with a totally geodesic hypersurface from which a given part of the sphere is equidistant.

Geodesic spheres in $\mathbb{H}^{n}$ do not touch the ideal boundary. They bound balls which cannot be foliated by spheres in codimension 1 which allows us to exclude them from the further analysis. From now on we shall study only unbounded totally umibilical hypersurfaces.

This makes sense to analyse totally umbilical hypersurfaces of $\mathbb{H}^{n}$ as well as codimension 1 totally umbilical foliations using only notions for spheres coming from $\Lambda^{n+1}$.

Choose a model for $\mathbb{H}^{n}$ as $e_{n+1}$. This oriented $(n-1)$-sphere could be treated as a unit ball. From Prop. 4 and its proof we obtain directly.

Corollary 6 Assume that $e_{n+1} \in \Lambda^{n+1}$ is a ball model of $\mathbb{H}^{n}$. An element $s \in \Lambda^{n+1}$ represents an unbounded totally umbilical hypersurface of $\mathbb{H}^{n}$ if $s_{n+1} \in[-1,1]$. Moreover, the element s represents:

1) a totally geodesic hypersurface if $s_{n+1}=0$,

2) a hypersphere meeting ideal boundary at angle $\alpha$ if $s_{n+1}= \pm \cos \alpha$,

3) a horosphere which is internally tangent to the ideal boundary if $s_{n+1}= \pm 1$.

Every unbounded totally umbilical hypersurface of $\mathbb{H}^{n}$ divides the hyperbolic space into two domains with nonempty ideal boundary. From this we conclude that every totally umbilical codimension 1 foliation of $\mathbb{H}^{n}$ has leaves entirely contained in spheres which form oneparameter family.

Corollary 7 Every totally umbilical codimension 1 foliation of $\mathbb{H}^{n}$ is represented by a curve $\Gamma: \square \rightarrow \Lambda^{n+1}$ which lies in the region bounded by hyperplanes $x_{n+1}= \pm 1$.

Denote by $\Lambda_{0}^{n+1}=\Lambda^{n+1} \cap\left\{x_{n+1}=0\right\}$ the set of totally geodesic hypersurfaces and by $\Lambda_{1}^{n+1}=\Lambda^{n+1} \cap\left\{x_{n+1}=1\right\}$ 
- the set of horospheres. It is easy to see that $\Lambda_{0}^{n+1}$ is simply $n$-dimensional de Sitter space $\Lambda^{n}$ while $\Lambda_{1}^{n+1}$ is the light cone corresponding to $\mathbb{L}^{n+1}$ with the vertex $e_{n+1}$. Similarly, the set of totally umbilical hypersurfaces with the mean curvature equal to $\cos \alpha$ is rescaled $n$ dimensional de Sitter space given by $\langle x \mid x\rangle=\sin ^{2} \alpha$ for the first $n+1$ coordinates.

The converse to Cor. 7 is obviously not true. Some local conditions are studied by Langevin and the first author in [7]. More global conditions were found for totally geodesic foliations (see e.g. Figure 2).

Theorem 8 (Cz, Langevin [7]) Any unbounded curve $\Gamma: \mathbb{R} \rightarrow \Lambda_{0}^{n+1}$ such that

$$
\left|\left\langle\Gamma\left(t_{1}\right) \mid \Gamma\left(t_{2}\right)\right\rangle\right| \geq 1 \text { for all } t_{1}, t_{2}
$$

represents a totally geodesic codimension 1 foliation of $\mathbb{H}^{n}$. Moreover, any such foliation is transversely oriented and is represented by a curve $\Gamma$ as above.

The proof uses mainly the fact that spheres orthogonal to a given sphere are disjoint inside this sphere if they are at most tangent on the given sphere.

The above works even for $C^{0}$ foliations. For differentiable case we have the following

Corollary 9 An unbounded $C^{1}$ curve $\Gamma: \mathbb{R} \rightarrow \Lambda_{0}^{n+1}$ represents a totally geodesic codimension 1 foliations of $\mathbb{H}^{n}$ if $\Gamma$ is time-or-light-like i.e. $\left\langle\Gamma^{\prime}(t) \mid \Gamma^{\prime}(t)\right\rangle \leq 0$ for any $t$.

Strictly metrical characterization of totally umbilical foliations of $\mathbb{H}^{n}$ were described by Lużyńczyk and the first author in [8] under assumption that an orthogonal transversal is a geodesic.

Example 10 Well known examples of totally umbilical foliations of $\mathbb{H}^{n}$ are those by horospheres of the same end, totally geodesic orthogonal to a given geodesic or to a horocycle.

1) A light ray of the shifted light cone $\Lambda_{1}^{n+1}$ repre-

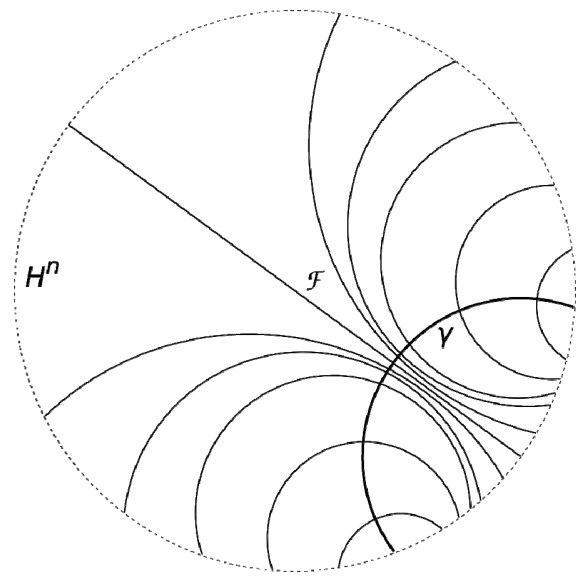

Figure 2. Totally geodesic foliation $\mathcal{F}$ orthogonal to a geodesic $\gamma$.

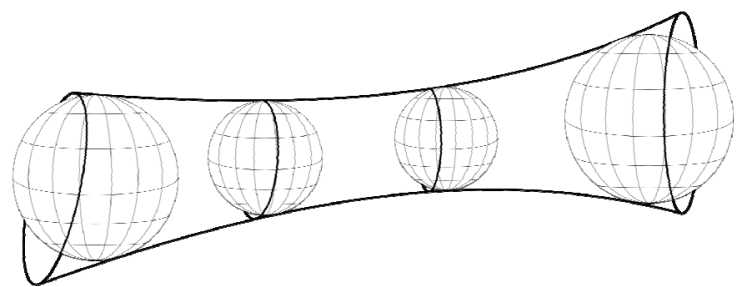

Figure 3. A canal surface with its spheres.

sents a foliation by horospheres with the same endpoint on the ideal boundary.

2) The intersection of de Sitter $n$-space $\Lambda_{0}^{n+1}$ with the time-like plane $x_{2}=\cdots=x_{n}=x_{n+1}=0$ represents a totally geodesic foliation orthogonal to a geodesic.

3) The intersection of de Sitter $n$--space $\Lambda_{0}^{n+1}$ with the space-like plane $x_{0}=x_{3}=\cdots=x_{n}=x_{n+1}=0$ represents a totally geodesic foliation orthogonal to a horocycle.

\subsection{Foliatons by Surfaces}

Some of hypersurfaces are envelopes of families of spheres. In particular, envelopes of one-parameter families of 2-spheres are objects which are well understable. They are easily represented as curves in de Sitter space $\Lambda^{4}$.

Definition 11 An envelope of an one-parameter family of 2-spheres is called a canal surface.

If a canal surface is a common envelope of two distinct one-parameter families of spheres we say that this surface is a Dupin cyclide.

Typical examples of a canal surface are a torus, a round cylinder, a cone of revolution or more generally a regular surface of revolution. A conformal image of a cylinder with a family of generating spheres is shown on

\section{Figure 3.}

Dupin cyclides could characterized in another way as conformal images of tori, cylinders and cones.

In $[5,6]$ the authors characterized foliations of $\mathbb{S}^{3}$ with canal leaves proving that such foliation comes only from the Reeb foliation by inserting a torical region filled out by tori and cylinders.

Some sophisticated methods led Langevin and Pawel Walczak to the fact that foliating compact hyperbolic 3-manifold is impossible neither by umbilical nor Dupin leaves.

\section{REFERENCES}

[1] M. Czarnecki and P. Walczak, "Extrinsic Geometry of Foliations," World Scientific, Singapore, 2006, pp. 149167.

[2] M. Badura and M. Czarnecki, "Recent Progress in Geometric Foliation Theory," World Scientific, Singapore, 2013. 
[3] J. O'Hara, "Energy of Knots and Conformal Geometry," World Scientific, Singapore, 2003.

[4] R. Langevin and P. G. Walczak, "Conformal Geometry of Foliations," Geometriae Dedicata, Vol. 132, No. 1, 2008, pp. 135-178. doi:10.1007/s10711-007-9213-1.

[5] A. Bartoszek, R. Langevin and P. G. Walczak, "Special Canal Surfaces of $\mathbb{S}^{3}$," Bulletin of the Brazilian Mathematical Society New Series, Vol. 42, No. 2, 2011, pp. 301-320.
[6] R. Langevin and P. G. Walczak, "Canal Foliations of $\mathbb{S}^{3}$," Journal of the Mathematical Society of Japan, Vol. 64, No. 2, 2012, pp. 659-682. doi:10.2969/jmsj/06420659

[7] M. Czarnecki and R. Langevin, "Totally Umbilical Foliations on Hyperbolic Spaces," in Preparation.

[8] M. Czarnecki and M. Lużyńczyk, "Umbilical Routes along Gedesics in Hyperbolic Spaces," in Preparation. 